ORIGINAL ARTICLE

\section{Primary Prevention of Cardiovascular Disease with a Mediterranean Diet}

\author{
Ramón Estruch, M.D., Ph.D., Emilio Ros, M.D., Ph.D., Jordi Salas-Salvadó, M.D., Ph.D., \\ Maria-Isabel Covas, D.Pharm., Ph.D., Dolores Corella, D.Pharm., Ph.D., \\ Fernando Arós, M.D., Ph.D., Enrique Gómez-Gracia, M.D., Ph.D., \\ Valentina Ruiz-Gutiérrez, Ph.D., Miquel Fiol, M.D., Ph.D., José Lapetra, M.D., Ph.D., \\ Rosa Maria Lamuela-Raventos, D.Pharm., Ph.D., Lluís Serra-Majem, M.D., Ph.D., \\ Xavier Pintó, M.D., Ph.D., Josep Basora, M.D., Ph.D., Miguel Angel Muñoz, M.D., Ph.D., \\ José V. Sorlí, M.D., Ph.D., José Alfredo Martínez, D.Pharm, M.D., Ph.D., and \\ Miguel Angel Martínez-González, M.D., Ph.D., for the PREDIMED Study Investigators**
}

ABSTRACT

BACKGROUND

Observational cohort studies and a secondary prevention trial have shown an inverse association between adherence to the Mediterranean diet and cardiovascular risk. We conducted a randomized trial of this diet pattern for the primary prevention of cardiovascular events.

\section{METHODS}

In a multicenter trial in Spain, we randomly assigned participants who were at high cardiovascular risk, but with no cardiovascular disease at enrollment, to one of three diets: a Mediterranean diet supplemented with extra-virgin olive oil, a Mediterranean diet supplemented with mixed nuts, or a control diet (advice to reduce dietary fat). Participants received quarterly individual and group educational sessions and, depending on group assignment, free provision of extra-virgin olive oil, mixed nuts, or small nonfood gifts. The primary end point was the rate of major cardiovascular events (myocardial infarction, stroke, or death from cardiovascular causes). On the basis of the results of an interim analysis, the trial was stopped after a median follow-up of 4.8 years.

\section{RESULTS}

A total of 7447 persons were enrolled (age range, 55 to 80 years); 57\% were women. The two Mediterranean-diet groups had good adherence to the intervention, according to self-reported intake and biomarker analyses. A primary end-point event occurred in 288 participants. The multivariable-adjusted hazard ratios were 0.70 ( $95 \%$ confidence interval [CI], 0.54 to 0.92 ) and 0.72 ( $95 \% \mathrm{CI}, 0.54$ to 0.96 ) for the group assigned to a Mediterranean diet with extra-virgin olive oil (96 events) and the group assigned to a Mediterranean diet with nuts (83 events), respectively, versus the control group (109 events). No diet-related adverse effects were reported.

\section{CONCLUSIONS}

Among persons at high cardiovascular risk, a Mediterranean diet supplemented with extra-virgin olive oil or nuts reduced the incidence of major cardiovascular events. (Funded by the Spanish government's Instituto de Salud Carlos III and others; Controlled-Trials.com number, ISRCTN35739639.)
The authors' affiliations are listed in the Appendix. Address reprint requests to Dr. Estruch at the Department of Internal Medicine, Hospital Clinic, Villarroel 170, 08036 Barcelona, Spain, or at restruch@ clinic.ub.es, or to Dr. Martínez-González at the Department of Preventive Medicine and Public Health, Facultad de Medicina-Clínica Universidad de Navarra, Irunlarrea 1, 31008 Pamplona, Spain, or atmamartinez@unav.es.

*The PREDIMED (Prevención con Dieta Mediterránea) study investigators are listed in the Supplementary Appendix, available at NEJM.org.

Drs. Estruch and Martínez-González contributed equally to this article.

This article was published on February 25, 2013, at NEJM.org.

N EnglJ Med 2013.

DOI: 10.1056/NEJMoa1200303

Copyright @ 2013 Massachusetts Medical Society. 
T HE TRADITIONAL MEDITERRANEAN DIET is characterized by a high intake of olive oil, fruit, nuts, vegetables, and cereals; a moderate intake of fish and poultry; a low intake of dairy products, red meat, processed meats, and sweets; and wine in moderation, consumed with meals. ${ }^{1}$ In observational cohort studies ${ }^{2,3}$ and a secondary prevention trial (the Lyon Diet Heart Study), ${ }^{4}$ increasing adherence to the Mediterranean diet has been consistently beneficial with respect to cardiovascular risk. ${ }^{2-4} \mathrm{~A}$ systematic review ranked the Mediterranean diet as the most likely dietary model to provide protection against coronary heart disease. ${ }^{5}$ Small clinical trials have uncovered plausible biologic mechanisms to explain the salutary effects of this food pattern. ${ }^{6-9}$ We designed a randomized trial to test the efficacy of two Mediterranean diets (one supplemented with extra-virgin olive oil and another with nuts), as compared with a control diet (advice on a low-fat diet), on primary cardiovascular prevention.

\section{METHODS}

\section{STUDY DESIGN}

The PREDIMED trial (Prevención con Dieta Mediterránea) was a parallel-group, multicenter, randomized trial. Details of the trial design are provided elsewhere. ${ }^{10-12}$ The trial was designed and conducted by the authors, and the protocol was approved by the institutional review boards at all study locations. The authors vouch for the accuracy and completeness of the data and all analyses and for the fidelity of this report to the protocol, which is available with the full text of this article at NEJM.org.

Supplemental foods were donated, including extra-virgin olive oil (by Hojiblanca and Patrimonio Comunal Olivarero, both in Spain), walnuts (by the California Walnut Commission), almonds (by Borges, in Spain), and hazelnuts (by La Morella Nuts, in Spain). None of the sponsors had any role in the trial design, data analysis, or reporting of the results.

\section{PARTICIPANT SELECTION AND RANDOMIZATION}

Eligible participants were men ( 55 to 80 years of age) and women (60 to 80 years of age) with no cardiovascular disease at enrollment, who had either type 2 diabetes mellitus or at least three of the following major risk factors: smoking, hypertension, elevated low-density lipoprotein cholesterol levels, low high-density lipoprotein cholesterol levels, overweight or obesity, or a family history of premature coronary heart disease. Detailed enrollment criteria are provided in the Supplementary Appendix, available at NEJM .org. All participants provided written informed consent.

Beginning on October 1, 2003, participants were randomly assigned, in a 1:1:1 ratio, to one of three dietary intervention groups: a Mediterranean diet supplemented with extra-virgin olive oil, a Mediterranean diet supplemented with nuts, or a control diet. Randomization was performed centrally by means of a computer-generated random-number sequence.

\section{INTERVENTIONS AND MEASUREMENTS}

The dietary intervention ${ }^{8,10-13}$ is detailed in the Supplementary Appendix. The specific recommended diets are summarized in Table 1. Participants in the two Mediterranean-diet groups received either extra-virgin olive oil (approximately 1 liter per week) or $30 \mathrm{~g}$ of mixed nuts per day (15 g of walnuts, $7.5 \mathrm{~g}$ of hazelnuts, and $7.5 \mathrm{~g}$ of almonds) at no cost, and those in the control group received small nonfood gifts. No total calorie restriction was advised, nor was physical activity promoted.

For participants in the two Mediterraneandiet groups, dietitians ran individual and group dietary-training sessions at the baseline visit and quarterly thereafter. In each session, a 14-item dietary screener was used to assess adherence to the Mediterranean diet $^{8,14}$ (Table S1 in the Supplementary Appendix) so that personalized advice could be provided to the study participants in these groups.

Participants in the control group also received dietary training at the baseline visit and completed the 14-item dietary screener used to assess baseline adherence to the Mediterranean diet. Thereafter, during the first 3 years of the trial, they received a leaflet explaining the lowfat diet (Table S2 in the Supplementary Appendix) on a yearly basis. However, the realization that the more infrequent visit schedule and less intense support for the control group might be limitations of the trial prompted us to amend the protocol in October 2006. Thereafter, participants assigned to the control diet received personalized advice and were invited to group sessions with the same frequency and intensity as those in the Mediterranean-diet groups, with 
the use of a separate 9-item dietary screener (Table S3 in the Supplementary Appendix).

A general medical questionnaire, a 137-item validated food-frequency questionnaire, ${ }^{15}$ and the Minnesota Leisure-Time Physical Activity Questionnaire were administered on a yearly basis. ${ }^{10}$ Information from the food-frequency questionnaire was used to calculate intake of energy and nutrients. Weight, height, and waist circumference were directly measured. ${ }^{16}$ Biomarkers of compliance, including urinary hydroxytyrosol levels (to confirm compliance in the group receiving extra-virgin olive oil) and plasma alpha-linolenic acid levels (to confirm compliance in the group receiving mixed nuts), were measured in random subsamples of participants at 1,3 , and 5 years (see the Supplementary Appendix).

\section{END POINTS}

The primary end point was a composite of myocardial infarction, stroke, and death from cardiovascular causes. Secondary end points were stroke, myocardial infarction, death from cardiovascular causes, and death from any cause. We used four sources of information to identify end points: repeated contacts with participants, contacts with family physicians, a yearly review of medical records, and consultation of the National Death Index. All medical records related to end points were examined by the end-point adjudication committee, whose members were unaware of the study-group assignments. Only end points that were confirmed by the adjudication committee and that occurred between October 1 , 2003, and December 1, 2010, were included in the analyses. The criteria for adjudicating primary and secondary end points are detailed in the Supplementary Appendix.

\section{STATISTICAL ANALYSIS}

We initially estimated that a sample of 9000 participants would be required to provide statistical power of $80 \%$ to detect a relative risk reduction of $20 \%$ in each Mediterranean-diet group versus the control-diet group during a 4-year follow-up period, assuming an event rate of $12 \%$ in the control group..$^{10,17}$ In April 2008, on the advice of the data and safety monitoring board and on the basis of lower-than-expected rates of end-point events, the sample size was recalculated as 7400 participants, with the assumption of a 6-year follow-up period and underlying event rates of
Table 1. Summary of Dietary Recommendations to Participants in the Mediterranean-Diet Groups and the Control-Diet Group.

Food Goal

Mediterranean diet

Recommended

Olive oil*

$\geq 4$ tbsp/day

Tree nuts and peanuts $\uparrow$

$\geq 3$ servings/wk

Fresh fruits

$\geq 3$ servings/day

Vegetables

Fish (especially fatty fish), seafood

$\geq 2$ servings/day

Legumes

Sofritot:

$\geq 3$ servings/wk

$\geq 3$ servings/wk

White meat

$\geq 2$ servings/wk

Wine with meals (optionally, only for habitual drinkers)

Instead of red meat

$\geq 7$ glasses/wk

Discouraged

Soda drinks

$<$ l drink/day

Commercial bakery goods, sweets, and pastries』

$<3$ servings/wk

Spread fats

$<$ l serving/day

Red and processed meats

$<$ l serving/day

Low-fat diet (control)

Recommended

Low-fat dairy products

$\geq 3$ servings/day

Bread, potatoes, pasta, rice

$\geq 3$ servings/day

Fresh fruits

$\geq 3$ servings/day

Vegetables

Lean fish and seafood

$\geq 2$ servings/wk

$\geq 3$ servings/wk

Discouraged

Vegetable oils (including olive oil)

$\leq 2$ tbsp/day

Commercial bakery goods, sweets, and pastries』

Nuts and fried snacks

$\leq$ l serving/wk

Red and processed fatty meats

$\leq 1$ serving / wk

Visible fat in meats and soups 9

$\leq l$ serving/wk

Fatty fish, seafood canned in oil

Always remove

Spread fats

$\leq$ l serving/wk

$\leq$ l serving/wk

Sofrito

$\leq 2$ servings/wk

* The amount of olive oil includes oil used for cooking and salads and oil consumed in meals eaten outside the home. In the group assigned to the Mediterranean diet with extra-virgin olive oil, the goal was to consume $50 \mathrm{~g}$ (approximately 4 tbsp) or more per day of the polyphenol-rich olive oil supplied, instead of the ordinary refined variety, which is low in polyphenols.

$\uparrow$ For participants assigned to the Mediterranean diet with nuts, the recommended consumption was one daily serving ( $30 \mathrm{~g}$, composed of $15 \mathrm{~g}$ of walnuts, $7.5 \mathrm{~g}$ of almonds, and $7.5 \mathrm{~g}$ of hazelnuts).

tSofrito is a sauce made with tomato and onion, often including garlic and aromatic herbs, and slowly simmered with olive oil.

$\int$ Commercial bakery goods, sweets, and pastries (not homemade) included cakes, cookies, biscuits, and custard.

I Participants were advised to remove the visible fat (or the skin) of chicken, duck, pork, lamb, or veal before cooking and the fat of soups, broths, and cooked meat dishes before consumption. 
$8.8 \%$ and $6.6 \%$ in the control and intervention groups, respectively. Power curves under several assumptions can be found in Figure S1 in the Supplementary Appendix.

Yearly interim analyses began after a median of 2 years of follow-up. With the use of O'BrienFleming stopping boundaries, the $\mathrm{P}$ values for stopping the trial at each yearly interim analysis were $5 \times 10^{-6}, 0.001,0.009$, and 0.02 for benefit and $9 \times 10^{-5}, 0.005,0.02$, and 0.05 for adverse effects. ${ }^{18}$ The stopping boundary for the benefit of the Mediterranean diets with respect to the primary end point was crossed at the fourth interim evaluation; on July 22, 2011, the data and safety monitoring board recommended stopping the trial on the basis of end points documented through December 1, 2010.

All primary analyses were performed on an intention-to-treat basis by two independent analysts. Time-to-event data were analyzed with the use of Cox models with two dummy variables (one for the Mediterranean diet with extra-virgin olive oil and another for the Mediterranean diet with nuts) to obtain two hazard ratios for the comparison with the control group. To account for small imbalances in risk factors at baseline among the groups, Cox regression models were used to adjust for sex, age, and baseline risk factors. We tested the proportionality of hazards with the use of time-varying covariates. All analyses were stratified according to center. Prespecified subgroup analyses were conducted according to sex, age, body-mass index (BMI), cardiovascular-risk-factor status, and baseline adherence to the Mediterranean diet. Sensitivity analyses were conducted under several assumptions, including imputation of data for missing values and participants who dropped out (see the Supplementary Appendix).

\section{RESULTS}

\section{BASELINE CHARACTERISTICS OF THE STUDY PARTICIPANTS}

From October 2003 through June 2009, a total of 8713 candidates were screened for eligibility, and

\begin{tabular}{|c|c|c|c|}
\hline Characteristic & $\begin{array}{c}\text { Mediterranean } \\
\text { Diet with EVOO } \\
\quad(\mathrm{N}=2543)\end{array}$ & $\begin{array}{l}\text { Mediterranean } \\
\text { Diet with Nuts } \\
\quad(\mathrm{N}=2454)\end{array}$ & $\begin{array}{l}\text { Control Diet } \\
(\mathrm{N}=2450)\end{array}$ \\
\hline Female sex 一 no. $(\%) \dagger$ & $1493(58.7)$ & $1326(54.0)$ & $1463(59.7)$ \\
\hline Age - yrì & $67.0 \pm 6.2$ & $66.7 \pm 6.1$ & $67.3 \pm 6.3$ \\
\hline \multicolumn{4}{|l|}{ Race or ethnic group - no. (\%) } \\
\hline White, from Europe & $2470(97.1)$ & $2390(97.4)$ & $2375(96.9)$ \\
\hline Hispanic, from Central or South America & $35(1.4)$ & $29(1.2)$ & $38(1.6)$ \\
\hline Other & $38(1.5)$ & $35(1.4)$ & $37(1.5)$ \\
\hline \multicolumn{4}{|l|}{ Smoking status - no. (\%) } \\
\hline Never smoked & $1572(61.8)$ & $1465(59.7)$ & $1527(62.3)$ \\
\hline Former smoker & $618(24.3)$ & $634(25.8)$ & $584(23.8)$ \\
\hline Current smoker & $353(13.9)$ & $355(14.5)$ & $339(13.8)$ \\
\hline \multicolumn{4}{|l|}{ Body-mass indextr } \\
\hline Mean & $29.9 \pm 3.7$ & $29.7 \pm 3.8$ & $30.2 \pm 4.0$ \\
\hline$<25-$ no. $(\%)$ & $195(7.7)$ & $204(8.3)$ & $164(6.7)$ \\
\hline $25-30-$ no. $(\%)$ & $1153(45.3)$ & $1163(47.4)$ & $1085(44.3)$ \\
\hline$>30-$ no. $(\%)$ & $1195(47.0)$ & $1087(44.3)$ & $1201(49.0)$ \\
\hline Waist circumference $-\mathrm{cm}$ & $100 \pm 10$ & $100 \pm 11$ & $101 \pm 11$ \\
\hline Waist-to-height ratiot- $\int$ & $0.63 \pm 0.06$ & $0.63 \pm 0.06$ & $0.63 \pm 0.07$ \\
\hline Hypertension — no. (\%) 9 & $2088(82.1)$ & $2024(82.5)$ & $2050(83.7)$ \\
\hline Type 2 diabetes - no. (\%) †\| & $1282(50.4)$ & $1143(46.6)$ & $1189(48.5)$ \\
\hline Dyslipidemia — no. (\%)** & $1821(71.6)$ & $1799(73.3)$ & $1763(72.0)$ \\
\hline Family history of premature $\mathrm{CHD}-$ no. (\%) †† & $576(22.7)$ & $532(21.7)$ & $560(22.9)$ \\
\hline
\end{tabular}




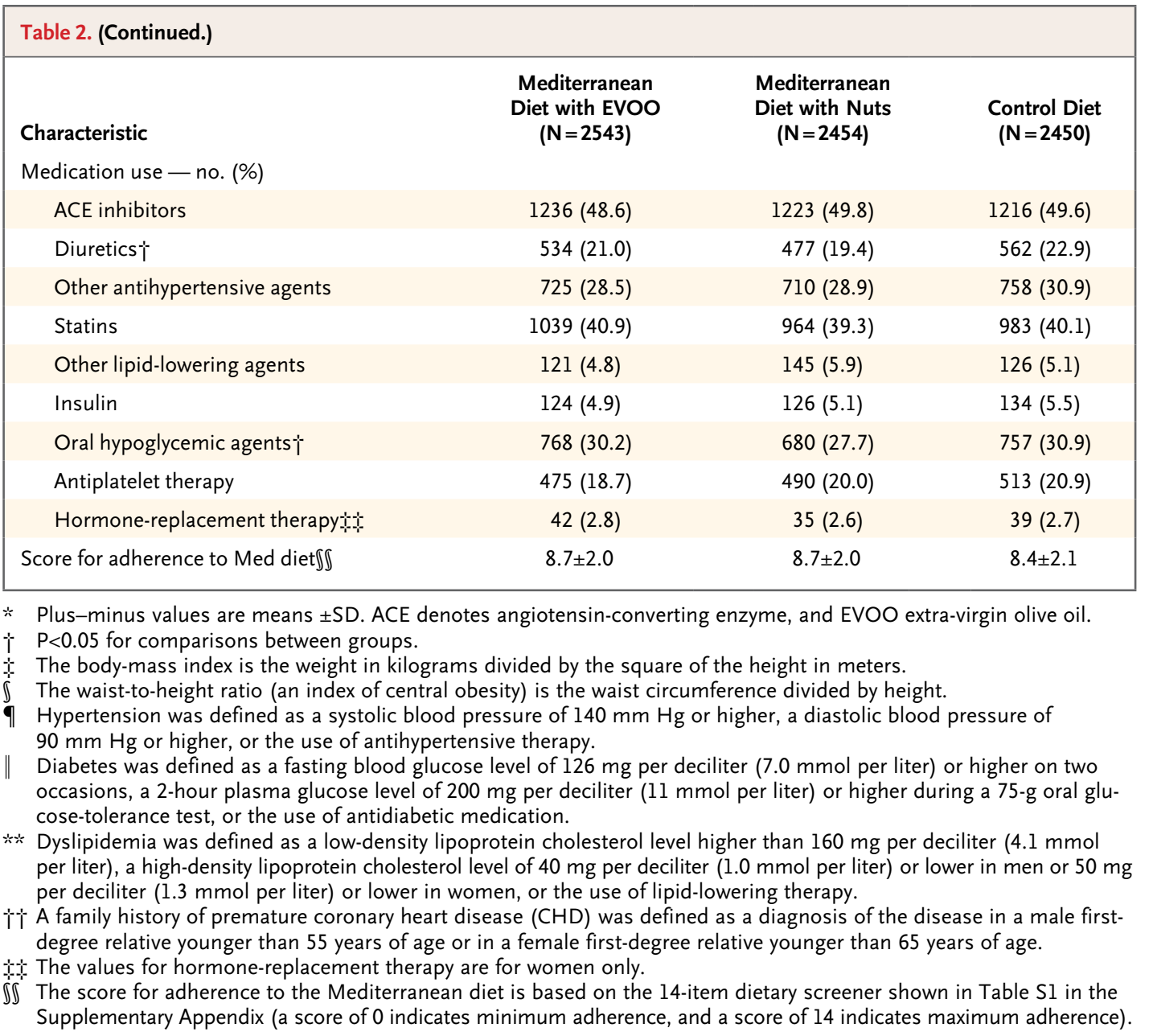

7447 were randomly assigned to one of the three study groups (Fig. S2 in the Supplementary Appendix). Their baseline characteristics according to study group are shown in Table 2. Drug-treatment regimens were similar for participants in the three groups, and they continued to be balanced during the follow-up period (Table S4 in the Supplementary Appendix).

Participants were followed for a median of 4.8 years (interquartile range, 2.8 to 5.8 ). After the initial assessment, 209 participants $(2.8 \%)$ chose not to attend subsequent visits, and their follow-up was based on reviews of medical records. By December 2010, a total of 523 participants $(7.0 \%)$ had been lost to follow-up for 2 or more years. Dropout rates were higher in the control group (11.3\%) than in the Mediterraneandiet groups (4.9\%) (Fig. S2 in the Supplementary Appendix). As compared with participants who remained in the trial, those who dropped out were younger (by 1.4 years), had a higher BMI (the weight in kilograms divided by the square of the height in meters; by 0.4 ), a higher waist-toheight ratio (by 0.01 ), and a lower score for adherence to the Mediterranean diet (by 1.0 points on the 14-item dietary screener) ( $\mathrm{P}<0.05$ for all comparisons).

\section{COMPLIANCE WITH THE DIETARY INTERVENTION}

Participants in the three groups reported similar adherence to the Mediterranean diet at baseline (Table 2, and Fig. S3 in the Supplementary Appendix) and similar food and nutrient intakes. During follow-up, scores on the 14-item Mediterranean-diet screener increased for the participants in the two Mediterranean-diet groups (Fig. S3 in the Supplementary Appendix). There were significant differences between these groups and the control group in 12 of the 14 items at 3 years (Table S5 in the Supplementary Appen- 
dix). Changes in objective biomarkers also indicated good compliance with the dietary assignments (Fig. S4 and S5 in the Supplementary Appendix).

Participants in the two Mediterranean-diet groups significantly increased weekly servings of fish (by 0.3 servings) and legumes (by 0.4 servings) in comparison with those in the control group (Table S6 in the Supplementary Appendix). In addition, participants assigned to a Mediterranean diet with extra-virgin olive oil and those assigned to a Mediterranean diet with nuts sig- nificantly increased their consumption of extravirgin olive oil (to 50 and $32 \mathrm{~g}$ per day, respectively) and nuts (to 0.9 and 6 servings per week, respectively). The main nutrient changes in the Mediterranean-diet groups reflected the fat content and composition of the supplemental foods (Tables S7 and S8 in the Supplementary Appendix). No relevant diet-related adverse effects were reported (see the Supplementary Appendix). We did not find any significant difference in changes in physical activity among the three groups.

\begin{tabular}{|c|c|c|c|c|c|}
\hline \multirow[t]{2}{*}{ End Point } & \multirow[t]{2}{*}{$\begin{array}{l}\text { Mediterranean } \\
\text { Diet with EVOO } \\
\quad(N=2543)\end{array}$} & \multirow[t]{2}{*}{$\begin{array}{l}\text { Mediterranean } \\
\text { Diet with Nuts } \\
\quad(\mathrm{N}=2454)\end{array}$} & \multirow[t]{2}{*}{$\begin{array}{l}\text { Control Diet } \\
(\mathrm{N}=\mathbf{2 4 5 0})\end{array}$} & \multicolumn{2}{|c|}{ P Value } \\
\hline & & & & $\begin{array}{l}\text { Mediterranean } \\
\text { Diet with EVOO } \\
\text { vs. Control Diet }\end{array}$ & $\begin{array}{c}\text { Mediterranean } \\
\text { Diet with Nuts } \\
\text { vs. Control Diet }\end{array}$ \\
\hline Person-yr of follow-up & 11,852 & 10,365 & 9763 & & \\
\hline \multicolumn{6}{|l|}{ Primary end point } \\
\hline No. of events & 96 & 83 & 109 & & \\
\hline Crude rate/ 1000 person-yr $(95 \% \mathrm{Cl})$ & $8.1(6.6-9.9)$ & $8.0(6.4-9.9)$ & $11.2(9.2-13.5)$ & 0.009 & 0.02 \\
\hline \multicolumn{6}{|l|}{ Secondary end points } \\
\hline \multicolumn{6}{|l|}{ Stroke } \\
\hline No. of events & 49 & 32 & 58 & & \\
\hline Crude rate/1000 person-yr $(95 \% \mathrm{Cl})$ & $4.1(3.1-5.5)$ & $3.1(2.1-4.4)$ & $5.9(4.5-7.7)$ & 0.03 & 0.003 \\
\hline \multicolumn{6}{|l|}{ Myocardial infarction } \\
\hline No. of events & 37 & 31 & 38 & & \\
\hline Crude rate/1000 person-yr $(95 \% \mathrm{Cl})$ & $3.1(2.2-4.3)$ & $3.0(2.0-4.2)$ & $3.9(2.8-5.3)$ & 0.31 & 0.25 \\
\hline \multicolumn{6}{|l|}{ Death from cardiovascular causes } \\
\hline No. of events & 26 & 31 & 30 & & \\
\hline Crude rate/1000 person-yr $(95 \% \mathrm{Cl})$ & $2.2(1.4-3.2)$ & $3.0(2.0-4.2)$ & $3.1(2.1-4.4)$ & 0.15 & 0.85 \\
\hline \multicolumn{6}{|l|}{ Death from any cause } \\
\hline No. of events & 118 & 116 & 114 & & \\
\hline Crude rate/1000 person-yr $(95 \% \mathrm{Cl})$ & $10.0(8.2-11.9)$ & $11.2(9.3-13.4)$ & $11.7(9.6-14.0)$ & 0.11 & 0.68 \\
\hline \multicolumn{6}{|l|}{$\begin{array}{l}\text { Hazard ratio for each Mediterranean diet } \\
\text { vs. control }(95 \% \mathrm{Cl})\end{array}$} \\
\hline \multicolumn{6}{|l|}{ Primary end point } \\
\hline Unadjusted & $0.70(0.53-0.91)$ & $0.70(0.53-0.94)$ & 1.00 (ref) & 0.009 & 0.02 \\
\hline Multivariable-adjusted $1 \mathbb{S}$ & $0.69(0.53-0.91)$ & $0.72(0.54-0.97)$ & 1.00 (ref) & 0.008 & 0.03 \\
\hline Multivariable-adjusted 29 & $0.70(0.54-0.92)$ & $0.72(0.54-0.96)$ & 1.00 (ref) & 0.01 & 0.03 \\
\hline \multicolumn{6}{|l|}{ Secondary end points\| } \\
\hline Stroke & $0.67(0.46-0.98)$ & $0.54(0.35-0.84)$ & 1.00 (ref) & 0.04 & 0.006 \\
\hline Myocardial infarction & $0.80(0.51-1.26)$ & $0.74(0.46-1.19)$ & 1.00 (ref) & 0.34 & 0.22 \\
\hline Death from cardiovascular causes & $0.69(0.41-1.16)$ & $1.01(0.61-1.66)$ & 1.00 (ref) & 0.17 & 0.98 \\
\hline Death from any cause & $0.82(0.64-1.07)$ & $0.97(0.74-1.26)$ & 1.00 (ref) & 0.15 & 0.82 \\
\hline
\end{tabular}




\begin{tabular}{|c|c|c|c|c|}
\hline Table 3. (Continued.) & & & & \\
\hline \multirow[t]{2}{*}{ End Point } & \multirow[t]{2}{*}{$\begin{array}{l}\text { Mediterranean } \\
\text { Diet with EVOO } \\
\qquad(\mathrm{N}=2543)\end{array}$} & \multirow[t]{2}{*}{$\begin{array}{l}\text { Mediterranean } \\
\text { Diet with Nuts } \\
(\mathrm{N}=2454)\end{array}$} & \multirow[t]{2}{*}{$\begin{array}{l}\text { Control Diet } \\
(\mathrm{N}=\mathbf{2 4 5 0})\end{array}$} & P Value ${ }^{\prime}$ \\
\hline & & & & $\begin{array}{cc}\text { Mediterranean } & \text { Mediterranean } \\
\text { Diet with EVOO } & \text { Diet with Nuts } \\
\text { vs. Control Diet } & \text { vs. Control Diet }\end{array}$ \\
\hline \multicolumn{5}{|l|}{$\begin{array}{c}\text { Hazard ratio for Mediterranean diets combined } \\
\text { vs. control }(95 \% \mathrm{Cl})\end{array}$} \\
\hline \multicolumn{5}{|l|}{ Primary end point } \\
\hline Unadjusted & \multicolumn{2}{|c|}{$0.70(0.55-0.89)$} & 1 (ref) & 0.003 \\
\hline Multivariable-adjusted $1 \mathbb{\int}$ & \multicolumn{2}{|c|}{$0.71(0.56-0.90)$} & 1 (ref) & 0.004 \\
\hline Multivariable-adjusted 2q & \multicolumn{2}{|c|}{$0.71(0.56-0.90)$} & 1 (ref) & 0.005 \\
\hline \multicolumn{5}{|l|}{ Secondary end points\| } \\
\hline Stroke & \multicolumn{2}{|c|}{$0.61(0.44-0.86)$} & 1 (ref) & 0.005 \\
\hline Myocardial infarction & \multicolumn{2}{|c|}{$0.77(0.52-1.15)$} & 1 (ref) & 0.20 \\
\hline Death from cardiovascular causes & \multicolumn{2}{|c|}{$0.83(0.54-1.29)$} & 1 (ref) & 0.41 \\
\hline Death from any cause & \multicolumn{2}{|c|}{$0.89(0.71-1.12)$} & 1 (ref) & 0.32 \\
\hline
\end{tabular}

* $\mathrm{Cl}$ denotes confidence interval, and ref reference.

$\dagger$ All $\mathrm{P}$ values were calculated with the use of Cox proportional-hazards models with robust variance estimators and stratification according to recruiting center.

$\checkmark$ The primary end point was a composite of myocardial infarction, stroke, and death from cardiovascular causes.

$\int$ The primary end point was stratified according to recruiting center and adjusted for sex, age (continuous variable), family history of premature coronary heart disease (yes or no), and smoking status (never smoked, former smoker, or current smoker).

१The primary end point was additionally adjusted for body-mass index (continuous variable), waist-to-height ratio (continuous variable), hypertension at baseline (yes or no), dyslipidemia at baseline (yes or no), and diabetes at baseline (yes or no).

$\|$ The secondary end points were stratified according to recruiting center and adjusted for sex, age (continuous variable), family history of premature coronary heart disease (yes or no), smoking status (never smoked, former smoker, or current smoker), body-mass index (continuous variable), waist-to-height ratio (continuous variable), hypertension at baseline (yes or no), dyslipidemia at baseline (yes or no), and diabetes at baseline (yes or no).

\section{END POINTS}

The median follow-up period was 4.8 years. A total of 288 primary-outcome events occurred: 96 in the group assigned to a Mediterranean diet with extra-virgin olive oil (3.8\%), 83 in the group assigned to a Mediterranean diet with nuts (3.4\%), and 109 in the control group (4.4\%). Taking into account the small differences in the accrual of person-years among the three groups, the respective rates of the primary end point were 8.1, 8.0, and 11.2 per 1000 person-years (Table 3). The unadjusted hazard ratios were 0.70 (95\% confidence interval [CI], 0.53 to 0.91 ) for a Mediterranean diet with extra-virgin olive oil and 0.70 (95\% CI, 0.53 to 0.94 ) for a Mediterranean diet with nuts (Fig. 1) as compared with the control diet $(\mathrm{P}=0.015$, by the likelihood ratio test, for the overall effect of the intervention).

The results of multivariate analyses showed a similar protective effect of the two Mediterranean diets versus the control diet with respect to the primary end point (Table 3). Regarding components of the primary end point, only the comparisons of stroke risk reached statistical significance (Table 3, and Fig. S6 in the Supplementary Appendix). The Kaplan-Meier curves for the primary end point diverged soon after the trial started, but no effect on all-cause mortality was apparent (Fig. 1). The results of several sensitivity analyses were also consistent with the findings of the primary analysis (Table S9 in the Supplementary Appendix).

\section{SUBGROUP ANALYSES}

Reductions in disease risk in the two Mediterranean-diet groups as compared with the control group were similar across the prespecified subgroups (Fig. 2, and Table S10 in the Supplementary Appendix). In addition, to account for the protocol change in October 2006 whereby the intensity of dietary intervention in the control group was increased, we compared hazard ratios 


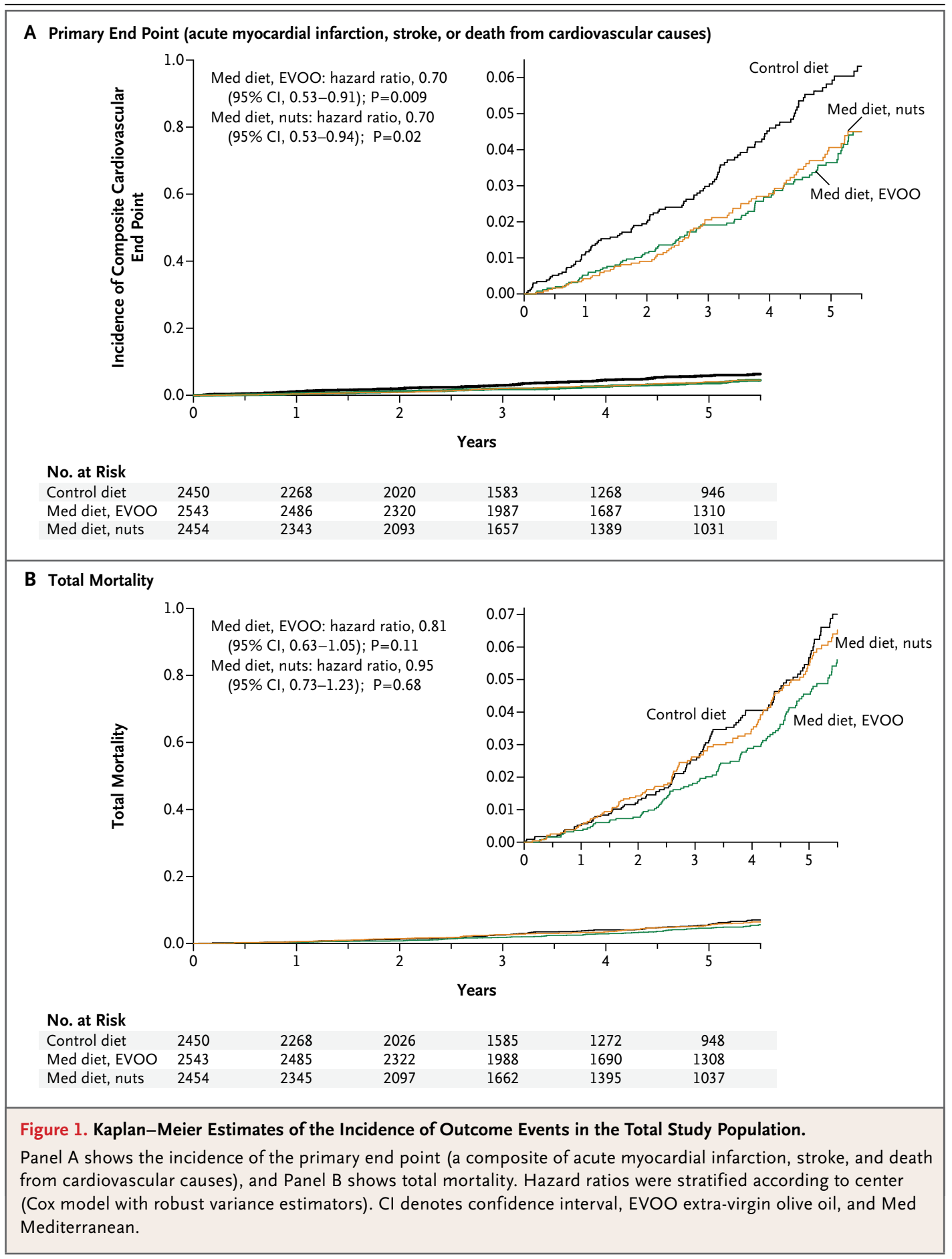

for the Mediterranean-diet groups (both groups merged vs. the control group) before and after this date. Adjusted hazard ratios were 0.77 (95\% CI, 0.59 to 1.00) for participants recruited before October 2006 and 0.49 (95\% CI, 0.26 to 0.92 ) for those recruited thereafter $(\mathrm{P}=0.21$ for interaction). duction of approximately 3 major cardiovascular

\section{DISCUSSION}

In this trial, an energy-unrestricted Mediterranean diet supplemented with either extra-virgin olive oil or nuts resulted in an absolute risk re- 


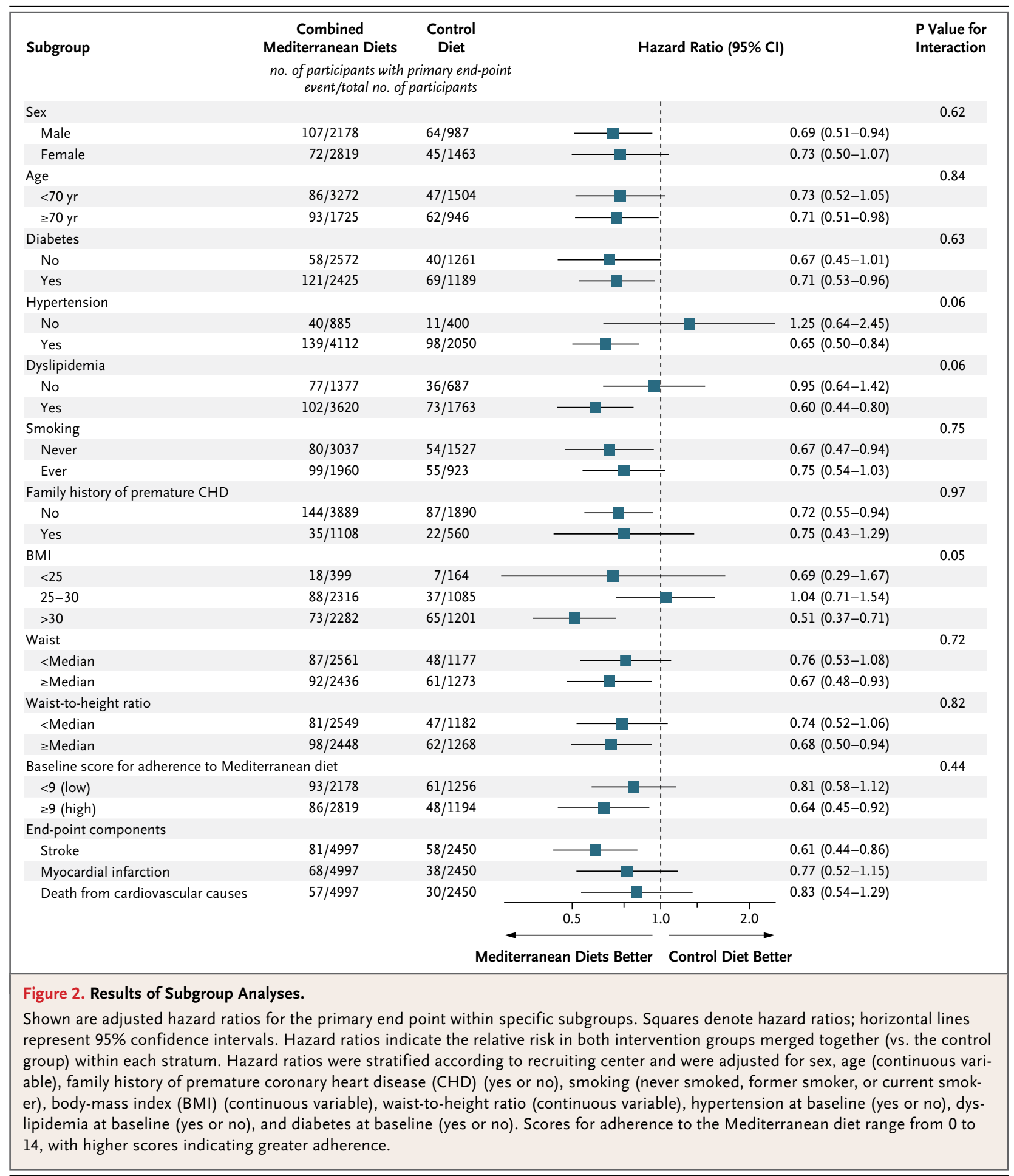

events per 1000 person-years, for a relative risk reduction of approximately $30 \%$, among highrisk persons who were initially free of cardiovascular disease. These results support the benefits of the Mediterranean diet for cardiovascular risk reduction. They are particularly relevant given the challenges of achieving and maintaining weight loss. The secondary prevention Lyon Diet Heart Study also showed a large reduction in rates of coronary heart disease events with a modified 
Mediterranean diet enriched with alpha-linolenic acid (a key constituent of walnuts). That result, however, was based on only a few major events. 4,19,20

There were small between-group differences in some baseline characteristics in our trial, which were not clinically meaningful but were statistically significant, and we therefore adjusted for these variables. In fully adjusted analyses, we found significant results for the combined cardiovascular end point and for stroke, but not for myocardial infarction alone. This could be due to stronger effects on specific risk factors for stroke but also to a lower statistical power to identify effects on myocardial infarction. Our findings are consistent with those of prior observational studies of the cardiovascular protective effects of the Mediterranean diet, ${ }^{2,5}$ olive oil, ${ }^{21-23}$ and nuts ${ }^{24,25}$; smaller trials assessing effects on traditional cardiovascular risk factors ${ }^{6-9}$ and novel risk factors, such as markers of oxidation, inflammation, and endothelial dysfunction ${ }^{6,8,26-28}$; and studies of conditions associated with high cardiovascular risk - namely, the metabolic syndrome ${ }^{6,16,29}$ and diabetes. ${ }^{30-32}$ Thus, a causal role of the Mediterranean diet in cardiovascular prevention has high biologic plausibility. The results of our trial might explain, in part, the lower cardiovascular mortality in Mediterranean countries than in northern European countries or the United States. ${ }^{33}$

The risk of stroke was reduced significantly in the two Mediterranean-diet groups. This is consistent with epidemiologic studies that showed an inverse association between the Mediterranean diet $^{2,34}$ or olive-oil consumption ${ }^{22}$ and incident stroke.

Our results compare favorably with those of the Women's Health Initiative Dietary Modification Trial, wherein a low-fat dietary approach resulted in no cardiovascular benefit. ${ }^{35}$ Salient components of the Mediterranean diet reportedly associated with better survival include moderate consumption of ethanol (mostly from wine), low consumption of meat and meat products, and high consumption of vegetables, fruits, nuts, legumes, fish, and olive oil. ${ }^{36,37}$ Perhaps there is a synergy among the nutrient-rich foods included in the Mediterranean diet that fosters favorable changes in intermediate pathways of cardiometabolic risk, such as blood lipids, insulin sensitivity, resistance to oxidation, inflammation, and vasoreactivity. ${ }^{38}$
Our study has several limitations. First, the protocol for the control group was changed halfway through the trial. The lower intensity of dietary intervention for the control group during the first few years might have caused a bias toward a benefit in the two Mediterranean-diet groups, since the participants in these two groups received a more intensive intervention during that time. However, we found no significant interaction between the period of trial enrollment (before vs. after the protocol change) and the benefit in the Mediterranean-diet groups. Second, we had losses to follow-up, predominantly in the control group, but the participants who dropped out had a worse cardiovascular risk profile at baseline than those who remained in the study, suggesting a bias toward a benefit in the control group. Third, the generalizability of our findings is limited because all the study participants lived in a Mediterranean country and were at high cardiovascular risk; whether the results can be generalized to persons at lower risk or to other settings requires further research.

As with many clinical trials, the observed rates of cardiovascular events were lower than anticipated, with reduced statistical power to separately assess components of the primary end point. However, favorable trends were seen for both stroke and myocardial infarction. We acknowledge that, even though participants in the control group received advice to reduce fat intake, changes in total fat were small and the largest differences at the end of the trial were in the distribution of fat subtypes. The interventions were intended to improve the overall dietary pattern, but the major between-group differences involved the supplemental items. Thus, extravirgin olive oil and nuts were probably responsible for most of the observed benefits of the Mediterranean diets. Differences were also observed for fish and legumes but not for other food groups. The small between-group differences in the diets during the trial are probably due to the facts that for most trial participants the baseline diet was similar to the trial Mediterranean diet and that the control group was given recommendations for a healthy diet, suggesting a potentially greater benefit of the Mediterranean diet as compared with Western diets.

In conclusion, in this primary prevention trial, we observed that an energy-unrestricted Mediterranean diet, supplemented with extra-virgin 
olive oil or nuts, resulted in a substantial reduction in the risk of major cardiovascular events among high-risk persons. The results support the benefits of the Mediterranean diet for the primary prevention of cardiovascular disease.

Supported by the official funding agency for biomedical research of the Spanish government, Instituto de Salud Carlos III (ISCIII), through grants provided to research networks specifically developed for the trial (RTIC G03/140, to Dr. Estruch; RTIC RD 06/0045, to Dr. Martínez-González and through Centro de Investigación Biomédica en Red de Fisiopatología de la Obesidad y Nutrición [CIBERobn]), and by grants from Centro Nacional de Investigaciones Cardiovasculares (CNIC 06/2007), Fondo de Investigación Sanitaria-Fondo Europeo de Desarrollo Regional (PI04-2239, PI 05/2584, CP06/00100, PI07/0240, PI07/1138, PI07/0954, PI 07/0473, PI10/01407, PI10/02658, PI11/01647, and P11/02505), Ministerio de Ciencia e Innovación (AGL-200913906-C02 and AGL2010-22319-C03), Fundación Mapfre 2010, Consejería de Salud de la Junta de Andalucía (PI0105/2007), Public Health Division of the Department of Health of the Autonomous Government of Catalonia, Generalitat Valenciana (ACOMP06109, GVACOMP2010-181, GVACOMP2011-151, CS2010AP-111, and CS2011-AP-042), and Regional Government of Navarra (P27/2011).

Dr. Estruch reports serving on the board of and receiving lecture fees from the Research Foundation on Wine and Nutrition (FIVIN); serving on the boards of the Beer and Health Foundation and the European Foundation for Alcohol Research (ERAB); receiving lecture fees from Cerveceros de España and Sanofi-Aventis; and receiving grant support through his institution from Novartis. Dr. Ros reports serving on the board of and receiving travel support, as well as grant support through his institution, from the California Walnut Commission; serving on the board of the Flora Foundation (Unilever); serving on the board of and receiving lecture fees from Roche; serving on the board of and receiving grant support through his institution from Amgen; receiving consulting fees from Damm and Abbott
Laboratories; receiving consulting fees and lecture fees, as well as grant support through his institution, from Merck; receiving lecture fees from Danone, Pace, AstraZeneca, and Rottapharm; receiving lecture fees and payment for the development of educational presentations, as well as grant support through his institution, from Ferrer; receiving payment for the development of educational presentations from Recordati; and receiving grant support through his institution from Sanofi-Aventis, Takeda, Daiichi Sankyo, Nutrexpa, Feiraco, Unilever, and Karo Bio. Dr. Salas-Salvadó reports serving on the board of and receiving grant support through his institution from the International Nut and Dried Fruit Council; receiving consulting fees from Danone; and receiving grant support through his institution from Eroski and Nestlé. Dr. Arós reports receiving payment for the development of educational presentations from Menarini and AstraZeneca. Dr. Lamuela-Raventos reports serving on the board of and receiving lecture fees from FIVIN; receiving lecture fees from Cerveceros de España; and receiving lecture fees and travel support from PepsiCo. Dr. Serra-Majem reports serving on the boards of the Mediterranean Diet Foundation and the Beer and Health Foundation. Dr. Pintó reports serving on the board of and receiving grant support through his institution from the Residual Risk Reduction Initiative (R3i) Foundation; serving on the board of Omegafort; serving on the board of and receiving payment for the development of educational presentations, as well as grant support through his institution, from Ferrer; receiving consulting fees from Abbott Laboratories; receiving lecture fees, as well as grant support through his institution, from Merck and Roche; receiving lecture fees from Danone and Esteve; receiving payment for the development of educational presentations from Menarini; and receiving grant support through his institution from Sanofi-Aventis, Kowa, Unilever, Boehringer Ingelheim, and Karo Bio. No other potential conflict of interest relevant to this article was reported.

Disclosure forms provided by the authors are available with the full text of this article at NEJM.org.

We thank the participants in the trial for their enthusiastic and sustained collaboration and Joan Vila from Institut Municipal d'Investigació Mèdica, Barcelona, for expert assessment in the statistical analyses.

\section{APPENDIX}

The author's affiliations are as follows: Centro de Investigación Biomédica en Red de Fisiopatología de la Obesidad y Nutrición (R.E., E.R., J.S.-S., M.-I.C., D.C., M.F., J.L., R.M.L.-R., J.B., J.V.S., J.A.M.) and the PREDIMED (Prevención con Dieta Mediterránea) Network (RD 06/0045) (R.E., J.S.-S., F.A., E.G.-G., V.R.-G., R.M.L.-R., L.S.-M., X.P., J.B., J.V.S., J.A.M., M.A.M.-G.), Instituto de Salud Carlos III, Madrid; the Department of Internal Medicine (R.E.) and Lipid Clinic, Department of Endocrinology and Nutrition (E.R.), Institut d'Investigacions Biomèdiques August Pi I Sunyer, Hospital Clinic, University of Barcelona, Barcelona; Human Nutrition Department, Hospital Universitari Sant Joan, Institut d'Investigació Sanitaria Pere Virgili, Universitat Rovira i Virgili, Reus (J.S.-S.); Cardiovascular and Nutrition Research Group, Institut de Recerca Hospital del Mar, Barcelona (M.-I.C.); the Department of Preventive Medicine, University of Valencia, Valencia (D.C.); the Department of Cardiology, University Hospital of Alava, Vitoria (F.A.); the Department of Preventive Medicine, University of Malaga, Malaga (E.G.-G.); Instituto de la Grasa, Consejo Superior de Investigaciones Cientificas, Seville (V.R.-G.); Institute of Health Sciences (IUNICS), University of Balearic Islands, and Hospital Son Espases, Palma de Mallorca (M.F.); the Department of Family Medicine, Primary Care Division of Seville, San Pablo Health Center, Seville (J.L.); the Department of Nutrition and Food Science, School of Pharmacy, Xarxa de Referència en Tecnologia dels Aliments, Instituto de Investigación en Nutrición y Seguridad Alimentaria, University of Barcelona, Barcelona (R.M.L.-R.); the Department of Clinical Sciences, University of Las Palmas de Gran Canaria, Las Palmas (L.S.-M.); Lipids and Vascular Risk Unit, Internal Medicine, Hospital Universitario de Bellvitge, Hospitalet de Llobregat, Barcelona (X.P.); Primary Care Division, Catalan Institute of Health, Institut d'Investigació en Atenció Primària Jordi Gol, Tarragona-Reus (J.B.) and Barcelona (M.A.M.); Primary Care Division, Valencia Institute of Health, Valencia (J.V.S.); and the Departments of Nutrition and Food Sciences, Physiology and Toxicology (J.A.M.) and Preventive Medicine and Public Health (M.A.M.-G.), University of Navarra, Pamplona - all in Spain.

\section{REFERENCES}

1. Willett WC, Sacks F, Trichopoulou A, et al. Mediterranean diet pyramid: a cultural model for healthy eating. Am J Clin Nutr 1995;61:Suppl:1402S-1406S.

2. Sofi F, Abbate R, Gensini GF, Casini
A. Accruing evidence on benefits of adherence to the Mediterranean diet on health: an updated systematic review and meta-analysis. Am J Clin Nutr 2010;92: 1189-96.
3. Serra-Majem L, Roman B, Estruch R. Scientific evidence of interventions using the Mediterranean diet: a systematic review. Nutr Rev 2006;64:S27-S47.

4. de Lorgeril M, Salen P, Martin JL, 
Monjaud I, Delaye J, Mamelle N. Mediterranean diet, traditional risk factors, and the rate of cardiovascular complications after myocardial infarction: final report of the Lyon Diet Heart Study. Circulation 1999;99:779-85.

5. Mente A, de Koning L, Shannon HS, Anand SS. A systematic review of the evidence supporting a causal link between dietary factors and coronary heart disease. Arch Intern Med 2009;169:659-69.

6. Esposito K, Marfella R, Ciotola M, et al. Effect of a Mediterranean-style diet on endothelial dysfunction and markers of vascular inflammation in the metabolic syndrome: a randomized trial. JAMA 2004; 292:1440-6.

7. Vincent-Baudry S, Defoort C, Gerber $M$, et al. The Medi-RIVAGE study: reduction of cardiovascular disease risk factors after a 3-mo intervention with a Mediterranean-type diet or a low-fat diet. Am J Clin Nutr 2005;82:964-71.

8. Estruch R, Martínez-González MA, Corella D, et al. Effects of a Mediterranean-style diet on cardiovascular risk factors: a randomized trial. Ann Intern Med 2006;145:1-11.

9. Shai I, Schwarzfuchs D, Henkin Y, et al. Weight loss with a low-carbohydrate, Mediterranean, or low-fat diet. N Engl J Med 2008;359:229-41. [Erratum, N Engl J Med 2009;361:2681.]

10. Martínez-González MA, Corella D, Salas-Salvadó J, et al. Cohort profile: design and methods of the PREDIMED study. Int J Epidemiol 2012;41:377-85.

11. The PREDIMED Study (http://www .predimed.org).

12. The PREDIMED network (http://www predimed.es).

13. Zazpe I, Sanchez-Tainta A, Estruch R, et al. A large randomized individual and group intervention conducted by registered dieticians increased the adherence to Mediterranean-type diets: the PREDIMED study. J Am Diet Assoc 2008;108:1134-44. 14. Schröder H, Fitó M, Estruch R, et al. A short screener is valid for assessing Mediterranean diet adherence among older Spanish men and women. J Nutr 2011; 141:1140-5.

15. Fernández-BallartJD, Piñol JL, Zazpe I, et al. Relative validity of a semi-quantitative food-frequency questionnaire in an elderly Mediterranean population of Spain. Br J Nutr 2010;103:1808-16.

16. Salas-Salvadó J, Fernández-Ballart J,
Ros E, et al. Effect of a Mediterranean diet supplemented with nuts on metabolic syndrome status: one-year results of the PREDIMED randomized trial. Arch Intern Med 2008;168:2449-58.

17. ALLHAT Officers and Coordinators for the ALLHAT Collaborative Research Group. Major outcomes in moderately hypercholesterolemic, hypertensive patients randomized to pravastatin vs usual care: the Antihypertensive and Lipid-Lowering Treatment to Prevent Heart Attack Tria (ALLHAT-LLT). JAMA 2002;288:2998-3007. 18. O'Brien PC, Fleming TR. A multiple testing procedure for clinical trials. Biometrics 1979;35:549-56.

19. Kris-Etherton P, Eckel RH, Howard BV, St Jeor S, Bazzarre TL. Lyon Diet Heart Study: benefits of a Mediterranean-style, National Cholesterol Education Program/ American Heart Association step I dietary pattern on cardiovascular disease. Circulation 2001;103:1823-5.

20. de Lorgeril M, Renaud S, Mamelle N, et al. Mediterranean alpha-linolenic acidrich diet in secondary prevention of coronary heart disease. Lancet 1994;343:14549. [Erratum, Lancet 1995;345:738.]

21. Bendinelli B, Masala G, Saieva C, et al. Fruit, vegetables, and olive oil and risk of coronary heart disease in Italian women: the EPICOR Study. Am J Clin Nutr 2011; 93:275-83.

22. Samieri C, Féart C, Proust-Lima C, et al. Olive oil consumption, plasma oleic acid and stroke incidence: the Three-City Study. Neurology 2011;77:418-25.

23. Buckland G, Travier N, Barricarte A, et al. Olive oil intake and CHD in the European Prospective Investigation into Cancer and Nutrition Spanish cohort. Br J Nutr 2012;108:2075-82.

24. Kris-Etherton PM, Hu FB, Ros E, Sabaté J. The role of tree nuts and peanuts in the prevention of coronary heart disease multiple potential mechanisms. J Nutr 2008;138:1746S-1751S

25. Ros E, Tapsell LC, Sabaté J. Nuts and berries for heart health. Curr Atheroscler Rep 2010;12:397-406.

26. Fitó $M$, Guxens $M$, Corella D, et al. Effect of a traditional Mediterranean diet on lipoprotein oxidation: a randomized controlled trial. Arch Intern Med 2007; 167:1195-203

27. Mena MP, Sacanella E, Vázquez-Agell $\mathrm{M}$, et al. Inhibition of circulating immune cell activation: a molecular antiinflam- matory effect of the Mediterranean diet. Am J Clin Nutr 2009;89:248-56.

28. Fuentes F, López-Miranda J, Sánchez E, et al. Mediterranean and low-fat diets improve endothelial function in hypercholesterolemic men. Ann Intern Med 2001;134:1115-9.

29. Kastorini CM, Milionis HJ, Esposito K, Giugliano D, Goudevenos JA, Panagiotakos DB. The effect of Mediterranean diet on metabolic syndrome and its components: a meta-analysis of 50 studies and 534,906 individuals. J Am Coll Cardiol 2011;57:1299-313.

30. Esposito K, Maiorino MI, Ceriello A, Giugliano D. Prevention and control of type 2 diabetes by Mediterranean diet: a systematic review. Diabetes Res Clin Pract 2010;89:97-102.

31. Salas-Salvadó J, Bulló M, Babio N, et al. Reduction in the incidence of type 2 diabetes with the Mediterranean diet: results of the PREDIMED-Reus nutrition intervention randomized trial. Diabetes Care 2011;34:14-9.

32. Martínez-González MA, de la FuenteArrillaga C, Nuñez-Córdoba JM, et al. Adherence to Mediterranean diet and risk of developing diabetes: prospective cohort study. BMJ 2008;336:1348-51.

33. Müller-Nordhorn J, Binting S, Roll S, Willich SN. An update on regional variation in cardiovascular mortality within Europe. Eur Heart J 2008;29:1316-26.

34. Kastorini CM, Milionis HJ, Ioannidi A, et al. Adherence to the Mediterranean diet in relation to acute coronary syndrome or stroke nonfatal events: a comparative analysis of a case/case-control study. Am Heart J 2011;162:717-24.

35. Howard BV, Van Horn L, Hsia J, et al. Low-fat dietary pattern and risk of cardiovascular disease: the Women's Health Initiative Randomized Controlled Dietary Modification Trial. JAMA 2006;295:655-66. 36. Trichopoulou A, Bamia C, Trichopoulos D. Anatomy of health effects of Mediterranean diet: Greek EPIC prospective cohort study. BMJ 2009;338:b2337.

37. Buckland G, Mayén AL, Agudo A, et al. Olive oil intake and mortality within the Spanish population (EPIC-Spain). Am J Clin Nutr 2012;96:142-9.

38. Jacobs DR Jr, Gross MD, Tapsell LC. Food synergy: an operational concept for understanding nutrition. Am J Clin Nutr 2009;89:1543S-1548S.

Copyright (C) 2013 Massachusetts Medical Society. 